\title{
Filme
}

\author{
Mittwoch, den 14. April 1971, 14.30 bis 17.45 $\mathrm{Uhr}$ \\ Donnerstag, den 15. April, 9.00 bis 12.15 und 14.30 bis $17.45 \mathrm{Uhr}$ \\ Freitag, den 16. April, 9.00 bis 12.15 und 15.30 bis $18.00 \mathrm{Uhr}$
}

\section{Thorako-abdominales Aortenaneurysma mit Angina intestinalis und Wirbelarrosionen}

Film

\section{G. Heberer und D. SachweH-Köln}

Die seltenen Aneurysmen am thorako-abdominalen Aortenabschnitt IV sowie im Bereich der Abgänge von Eingeweide- und Nierenarterien sind meist luischer Genese. Während sich die Symptomatologie im allgemeinen nicht von derjenigen infrarenaler Bauchaortenaneurysmen unterscheidet, kann die Stenose oder der thrombotische Verschluß von am Aneurysmasack abgehenden Organarterien zusätzliche Beschwerden im Sinne einer Funktionsinsuffizienz verursachen.

Der Film handelt von einem 59jährigen Kranken, der mit den Symptomen einer Angina intestinalis mit heftigen Schmerzen im Abdomen nach der Nahrungsaufnahme und progredientem Gewichtsverlust zur stationären Aufnahme kam. Die angiographische Abklärung eines bei der klinischen Untersuchung gefundenen expansiv pulsierenden Tumors im Oberbauch zeigte am thorako-abdominalen Aortenabschnitt IV ein großes, sackförmiges Aneurysma mit vorwiegend dorsaler Ausdehnung, das zu ausgeprägten Wirbelarrosionen bei Th 10 und 11 geführt hatte. Truncus coeliacus und A. mesenterica sup. stellten sich nicht dar, die rechte und linke Nierenarterie waren dagegen nicht in das Aneurysma einbezogen. Die absolute Operationsindikation bestand aufgrund der Penetration des Aneurysmas mit Wirbelkörperarrosion sowie der Angina intestinalis.

Methode der Wahl bei der Behandlung des thorako-abdominalen Aortenaneurysmas ist das sog. Umwandlungsverfahren, das im Operationsfilm demonstriert wird: Nach antero-lateraler Thorakotomie im 7.ICR, Durchtrennung des linken Rippenbogens und oberer medianer Laparotomie erfolgte die Präparation des übermannsfaustgroßen Aneurysmas, das 3 QF oberhalb des Zwerchfells begann und dicht über den Nierenarterienabgängen endete. Der erkrankte Gefäßabschnitt wurde zunächst durch eine seitenständig mit der thorakalen und infrarenalen Aorta anastomosierte Dacronprothese von $18 \mathrm{~mm}$ Durchmesser ohne Zirkulationsunterbrechung umgangen. Nach Implantation zweier Prothesenarme von jeweils $8 \mathrm{~mm}$ Durchmesser in Höhe der Eingeweidearterien wurden Truncus coeliacus und A. mesenterica sup. jeweils mit dem entsprechenden Prothesenarm End-zu-End anastomosiert. Danach erfolgte die Resektion des Aneurysmas, das an der Hinterwand breit in die Wirbelkörper Th 10 und 11 penetriert war sowie der Nahtversehluß der beiden Aortenstümpfe.

Die 8 Wochen nach der Operation durchgeführte Cineangiographie von der linken A. axillaris aus demonstrierte gut durchgängige Aorten- und Eingeweidearterienprothesen mit regelrechten Anastomosenverhältnissen.

Heute, 11 Monate nach der Operation, ist der Patient beschwerdefrei, insbesondere werden keine Symptome im Sinne einer Angina intestinalis mehr angegeben. Der Patient hat inzwischen gegenüber dem präoperativen Körpergewicht $8 \mathrm{~kg} \mathrm{zu}$ genommen. 